\title{
The Economic and Epidemiological Impact of COVID-19 and Government Policies: Part 1
}

Matthew Famiglietti, Research Associate

Fernando Leibovici, Economist

S. economic activity contracted sharply during 2020 as COVID-19 spread and government introduced

policies aimed at curbing the virus. Understanding the impact of the pandemic on economic activity as well as the effectiveness and economic impact of health and containment policies has been a major challenge faced by policymakers. The fundamental issue is that economic activity, COVID-19's spread, and health and containment policies are interconnected-they simultaneously influence each other.

The COVID-19 pandemic had a significant impact on economic activity but generated only mild changes in health containment policies.

In a recent working paper, we develop a methodology to investigate the causal linkages among COVID-19's spread, health and containment policies, and economic activity. ${ }^{1}$ In this two-part essay, we present a nontechnical summary of our approach and main findings. In particular, Part I explains our methods and summarizes how COVID-19 has affected economic activity and government policies. Part II describes the effects of government policies on COVID-19's spread and economic activity.

Our estimation strategy identifies the roles of COVID19's spread and government policies on economic activity by comparing the value of exports across U.S. states. Exports are our measure of economic activity because their demand is largely determined abroad and not directly impacted by U.S. COVID-19 infection rates, government policies, or economic activity. In particular, we compare narrowly defined export transactions across states that differ in the intensity of, timing of, and response to the pandemic.

To illustrate, consider exports of bicycles from Texas and New York to France. Texas had less-stringent containment policies than New York. Now suppose that both states had a similar rate of COVID-19 hospitalizations and faced the same demand from France for bikes. Our approach would attribute any differences in the exports of bicycles to differences in containment policies. More broadly, our method identifies the relative impact of COVID-19's spread and government policies by generalizing the example to all goods exported by the United States, from all U.S. states.

We use data from March 2020 to November 2020 to capture the pandemic prior to the arrival of vaccines. To measure economic activity, we use exports data from the U.S. Census Bureau. To measure government policy responses to COVID-19, we use the containment and health index from the Oxford COVID-19 Government Response Tracker (OxCGRT). ${ }^{2}$ Finally, our primary measure of COVID-19's spread across states is the number of hospitalizations per million people, which we obtain from the COVID Tracking Project compiled by the Atlantic based on data from the Centers for Disease Control and Prevention and the Department of Health and Human Services. We use these data along with a statistical model to identify the causal linkages among COVID-19's spread, health containment policies, and economic activity.

Figure 1 plots COVID-19's spread (hospitalizations), health containment policies, and economic activity (exports) over time in response to an outbreak of COVID-19. To illustrate, we consider a doubling in COVID-19 hospitalizations. Panel A of Figure 1 illustrates this temporary increase, which rapidly declines over time.

Panel B of Figure 1 shows that this dramatic increase in COVID-19 hospitalizations mildly tightens health containment policies. In particular, the health and containment index only increases by 1 point. For context, note that the index is on a scale of $0-100$ and its average value across states during the pandemic was 49 , remaining between 33 and 65 in most states over this period. This very modest policy response to the pandemic captures how U.S. states typically responded to the pandemic in the United States: The average state did not tighten health containment policies significantly in response to COVID-19 outbreaks.

Panel C of Figure 1 shows that COVID-19 outbreaks immediately reduce economic activity. Approximately three months after a doubling of hospitalizations, we estimate 
Figure 1

Shock to Hospitalizations

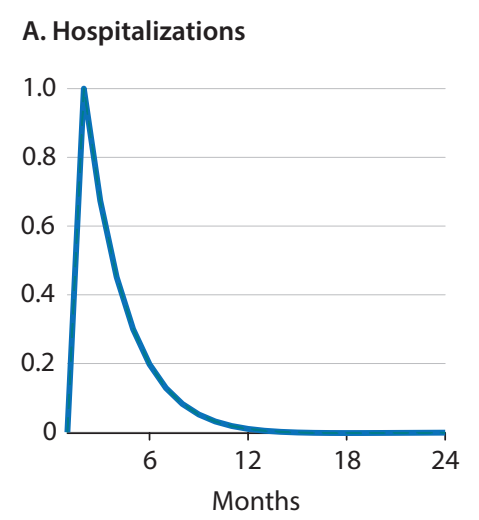

B. Health containment policies

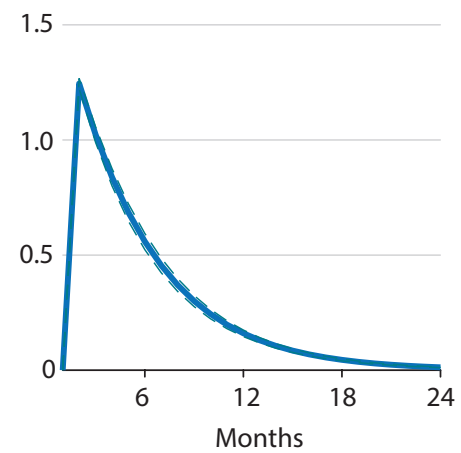

C. Exports

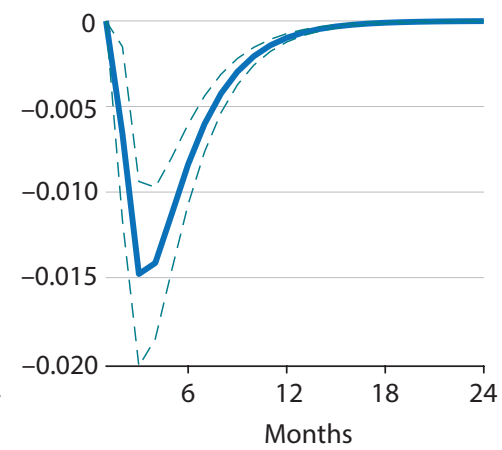

NOTE: Hospitalizations are measured per million people in logs, health and containment policies are measured using OxCGRT's index, and exports are measured as year-to-year log changes. The $y$-axes denote changes relative to pre-shock values of the respective variable. Dashed lines are 95 percent confidence intervals.

that exports decline by approximately 1.5 percent relative to the previous year. Additionally, this effect of COVID-19's spread is persistent, lasting around a year. This is a significant decline of economic activity, particularly in sectors that have been less affected by the pandemic than more contact-intensive sectors such as services.

In summary, the COVID-19 pandemic had a significant impact on economic activity but generated only mild changes in health containment policies. In Part 2 of this series, we summarize our findings on the impact of health containment and economic policies on economic activity and COVID-19's spread.

\begin{abstract}
Notes
1 Famiglietti, Matthew and Leibovici, Fernando. "The Impact of Health and Economic Policies on the Spread of COVID-19 and Economic Activity." Working Paper 2021-005, Federal Reserve Bank of St. Louis, 2021; https://research.stlouisfed.org/wp/more/2021-005.
\end{abstract}

2 See https://www.bsg.ox.ac.uk/research/research-projects/covid-19-government-response-tracker. 